\title{
Visual search and visual discomfort
}

\author{
Louise O'Hare, Alasdair D Clarke, Paul B Hibbard \\ Vision Lab, School of Psychology and Neuroscience, University of St Andrews, St Mary's \\ College, South Street, St Andrews KY16 9JP, Scotland, UK; e-mail: lo26@st-andrews.ac.uk \\ Received 27 February 2012, in revised form 22 November 2012
}

\begin{abstract}
Certain visual stimuli evoke perceptions of discomfort in non-clinical populations. We investigated the impact of stimuli previously judged as uncomfortable by non-clinical populations on a visual search task. One stimulus that has been shown to affect discomfort judgments is noise that has been filtered to have particular statistical properties (Juricevic et al, 2010 Perception 39 884-899). A second type of stimulus associated with visual discomfort is striped patterns (Wilkins et al, 1984 Brain 107989-1017). These stimuli were used as backgrounds in a visual search task, to determine their influence on search performance. Results showed that, while striped backgrounds did have an impact on visual search performance, this depended on the similarity between the target and background in orientation and spatial frequency. We found no evidence for a more generalised effect of discomfort on performance.
\end{abstract}

Keywords: visual search, visual discomfort, $1 / f$ noise

\section{Introduction}

\subsection{Visual discomfort}

Visual discomfort is defined as the unpleasant effects reported by some people whilst viewing particular stimuli. These stimuli include high-contrast striped patterns (Wilkins et al 1984), small font text, flickering lights (Sheedy et al 2003), and abstract images with particular spatial properties, such as some kinds of filtered noise patterns (Fernandez and Wilkins 2008; Juricevic et al 2010). Reported effects include distortions, illusions, nausea, headache (Wilkins et al 1984), blurred vision, and pain around the eyes (Sheedy et al 2003).

Clinical populations, such as migraineurs and people with epilepsy, have reported similar, but more extreme, responses on viewing such stimuli compared to non-clinical populations. For example, high-contrast striped patterns have been shown to increase the probability of epileptogenic activity in those susceptible (Wilkins et al 1979), and can even elicit seizures (Radhakrishnan et al 2005). Migraine sufferers tend to be susceptible to migraine attacks when viewing similar types of stimuli (eg Marcus and Soso 1989). Aurora and Wilkinson (2007) suggested that these populations may have excessive cortical responses. This predisposition to excessive responses could exacerbate the problem of difficult stimuli and therefore result in more discomfort in these groups.

Whilst the viewing of high-contrast gratings might have objectively measurable consequences in clinical populations, visual discomfort itself is, by definition, subjective (Lambooij et al 2011). The purpose of the current investigation is not to measure perceived visual discomfort, but instead to ascertain whether stimuli previously identified as uncomfortable, in both the clinical and non-clinical literature, affect performance on visual tasks.

\subsection{Impact of visual discomfort}

There is existing evidence that perceived visual discomfort could affect visual performance in general populations. Much of the previous evidence concerns individuals who score highly on measures of visual discomfort susceptibility, such as the Visual Discomfort Scale, or VDS (Conlon et al 1999). Conlon et al (2001) found that high scorers on this scale have 
poorer contrast discrimination thresholds than low scorers. Conlon and Humphreys (2001) found that high visual discomfort sufferers showed longer reaction times than controls on a pop-out visual search task, when the target was of a different orientation than the distractors. Additionally, high scorers on the VDS showed more of a detriment to performance with increasing set size. This indicates that the detriment was not a simple overall slowing for this group, but an interaction with the stimulus.

As well as affecting low-level visual tasks, there is some evidence that susceptibility to visual discomfort might have an impact on everyday tasks: individuals with high scores on the VDS are associated with lower academic achievement compared to low scorers (Chase et al 2009). Additionally, those with high scores on another scale of visual discomfort, the Pattern Glare Test, have also shown a reduced rate of reading compared to low scorers (Allen et al 2008). Although individual differences are not the subject of this study, this research demonstrates that those perceiving more discomfort show poorer visual task performance, in samples drawn from the general population.

\subsection{Stimulus properties increase perceived discomfort}

The amount of discomfort experienced when viewing stimuli can be influenced by their spatial attributes. For example, discomfort judgments of striped patterns are affected by the extent of the pattern, length of the stripes, duty cycle, and spatial frequency (Wilkins et al 1984). It might be expected that manipulations that increase perceived discomfort, such as changing the spatial frequency content of either noise patterns or striped gratings, would have a detrimental effect on performance in visual tasks.

For example, the global stimulus configuration can be an important factor: chequerboard stimuli have been shown to be less problematic than gratings for epilepsy sufferers (Wilkins et al 1975). Also, a shorter aspect ratio of stripes, making them more like chequerboard patterns, has been shown to produce less perceived discomfort in non-clinical populations (Wilkins et al 1984). Manipulations of global configuration have also been found to affect performance on visual search tasks. Conlon et al (1998) investigated reaction times in a task where subjects were instructed to count the numbers of letters E. The letters were presented so that their global configuration was plaid-, chequerboard-, or square-wave-like. Observers showed longer reaction times when the stimuli were presented in a square-wave-like pattern compared to the chequerboard- and plaid-like configurations. Additionally, high scorers on the VDS were also found to show slower reaction times on a visual search task than low or moderate scorers on this task. These results suggest that the longer reaction times found for square-wave-like configurations result from the visual discomfort associated with these stimuli, and this is more so for those reporting susceptibility to visual discomfort.

Similarly, spatial frequency is another stimulus property affecting visual performance that is also associated with discomfort. Gratings with a spatial frequency of 1 to 4 cycles deg-1 have previously been shown to increase epileptiform activity in epilepsy sufferers (Wilkins et al 1979) and discomfort in non-clinical populations (Wilkins et al 1984), compared to other spatial frequencies. Spatial frequency manipulations have been shown to affect discrimination of letters hidden against a striped background (Chronicle and Wilkins 1996). Additionally, Conlon and Hine (2000) used an oblique line target amongst vertical distractors to investigate visual search performance in high and low discomfort groups. Both high and low discomfort groups showed increased reaction time when a striped background was present. The 2 cycles $\mathrm{deg}^{-1}$ background resulted in a greater detriment to performance than the 15 cycles deg-1 background. As the 2 cycles deg $^{-1}$ stimulus is in the range more associated with visual discomfort than the 15 cycles $\mathrm{deg}^{-1}$ stimulus (Wilkins et al 1984), this result could be interpreted as an effect of visual discomfort on reaction times in visual search tasks. 
In the case of noise patterns, discomfort judgments might relate to global image statistics. Excess contrast energy at particular spatial frequencies has also been shown to increase discomfort judgments (Fernandez and Wilkins 2008; Juricevic et al 2010; O'Hare and Hibbard 2011). Juricevic et al (2010) have suggested that these stimuli are perceived as uncomfortable as their image statistics are different from those that typify natural images. Researchers have proposed that the visual system is optimised to efficiently code images with the statistics typically found in natural images (Field 1987, 1994; Simoncelli and Olshausen 2001). It follows that images with statistics differing from those typical of natural images would result in inefficient neural responses, with an elevated metabolic cost (Barlow 1961). One typical characteristic of natural images is an amplitude spectrum characterised as $1 / f^{\beta}$, where $f$ is spatial frequency and $\beta$ varies between 0.8 and 1.5 (Tolhurst et al 1992). Juricevic et al (2010) demonstrated that manipulating the slope of the amplitude spectrum (varying the value of $\beta$ ) affected discomfort judgments in both filtered-noise and 'Mondrian' patterns, comprised of randomly positioned, overlapping coloured rectangles. Exponent values closer to those of typical natural images $(\beta=1)$ were found to be judged as more comfortable than those with steeper slopes ( $\beta$ values closer to 2 ). This is consistent with other evidence that manipulating the amplitude spectrum of noise patterns affects discomfort judgments (Fernandez and Wilkins 2008; O'Hare and Hibbard 2011).

\subsection{Measures of performance}

Visual search was chosen as the task in the current experiment. It is important to investigate all aspects of performance, as an effect on reaction time alone has not been universally found in previous studies investigating the relationship between search performance and visual discomfort. For example, Allen et al (2008) measured search performance of high scorers on another measure of visual discomfort, the Pattern Related Visual Stress (PRVS) score. They found no difference in reaction time, but there was an increased error rate in the highscoring group compared to the low-scoring group. This study used a visual search task that involved counting the number of a particular digit. This target was presented in a window surrounded by an outer background of letters. This result suggests that the presence of a periodic background may detrimentally affect search performance, and that it is important to take account of both reaction time and error rate when assessing performance. Allen et al (2008) ascribe the increased error rate to discomfort. However, alternative possible explanations that could also account for this include crowding (Leat et al 1999) or distraction, due to the similarity of the target and background (Lichtenstein-Vidne et al 2007).

\section{$1.5 \mathrm{Aim}$}

The aim of the current study is to investigate visual search performance against search areas identified by previous research as being comfortable and uncomfortable. We hypothesise that uncomfortable patterns will detrimentally affect visual search performance compared to a more comfortable stimulus. Overall, we expect poorer performance for uncomfortable stimuli. We also make a more specific prediction: if stimuli previously identified as uncomfortable are aversive, we might expect observers to adopt a search strategy that minimises the time for which they are viewed. Thus observers might abandon the search more quickly against uncomfortable, compared to comfortable backgrounds. The effects of visual discomfort are particularly relevant to visual tasks that require prolonged viewing, since discomfort tends to build up over time (eg Chase et al 2009). Thus, it is hypothesised that increased discomfort would decrease motivation to continue to search for an extended period of time. The critical condition in our study is the target-absent condition: when the target is not present, we predict shorter reaction times, as observers are less inclined to continue to look at the uncomfortable background as they carry out their search. 


\section{Experiment 1: Filtered noise backgrounds}

In experiment $1,1 / f^{\beta}$ filtered noise patterns were used as the search areas, where $\beta=1$ has been shown to be judged as more comfortable, and $\beta=2$ as being less comfortable (Juricevic et al 2010). Experiment 1 consisted of two parts. The first part of the experiment determined $75 \%$ correct contrast detection thresholds in order to provide a performance-based metric to match task difficulty against the two noise search areas, when viewing time was fixed. A short ( $2 \mathrm{~s})$ and a long (10 s) presentation time were used, in order to obtain two levels of difficulty for the subsequent search task. The second part of the experiment involved a visual search task against filtered noise search areas (see figure 1 for an example of the stimuli). There were three target conditions: easy, hard, and absent; and two search area conditions: $\beta=1$ or 2 . The easy target-present (TP) condition used the target contrast found for the $2 \mathrm{~s}$ threshold experiment in the preceding part of the experiment. Similarly, the hard TP condition used the results of the $10 \mathrm{~s}$ threshold experiment. The critical comparison involves the target-absent trials: it was predicted that observers would quit searching faster against uncomfortable search areas compared to comfortable search areas.

\subsection{Method}

2.1.1 Apparatus. Stimuli were presented on a 20 inch Iiyama HM204DT A Diamontron V3-CRT Vision Master monitor, which was calibrated with a Minolta LS-110 photometer. The refresh rate was $100 \mathrm{~Hz}$, and the resolution was $1280 \times 1024$ pixels. Observers were at a distance of $50 \mathrm{~cm}$, meaning that the $1024 \times 1024$ pixel search background image subtended approximately $31^{\circ}$. Luminance range was between 1.35 and $34.66 \mathrm{~cd} \mathrm{~m}^{-2}$. An Eyelink 1000 (SR Research Systems) eye tracker was used during the visual search part of experiment 1 . Software from the Eyelink Toolbox Version 1.4.4 (Cornelissen et al 2002) was used to operate the hardware.

2.1.2 Observers. Eight observers (mean age $=24.5$ years, $\mathrm{SD}=1.20$ years), who were naive to the purposes of the experiment, participated in the first part of the study. All observers had either normal vision, or were corrected to normal with the use of contact lenses. All experiments were approved by the University of St Andrews Teaching and Research Ethics Committee.

2.1.3 Stimuli. Stimuli were created using MATLAB 2006b (The Mathworks Ltd). $1 / f^{\beta}$ filtered noise images were created with $\beta=1$ or 2 search backgrounds. These backgrounds have previously been shown to be comfortable and uncomfortable, respectively (Juricevic et al 2010). They were created by filtering Gaussian white noise in the Fourier frequency domain. Examples of the stimulus backgrounds are presented in figure 1. The target consisted of a vertical Gabor patch ( 3 cycles deg ${ }^{-1}, \sigma=0.26 \mathrm{deg}$ ). The target was presented at a random location on the screen, but avoiding a central strip of $2 \mathrm{deg}$, and a 2 deg strip from the extreme edge of the top and bottom of the screen, and a $1 \mathrm{deg}$ strip from the left and the right extremes. Initial fixations were encouraged to be in the centre of the screen by the addition of a white fixation cross in between each trial.

2.1.4 Procedure: Contrast threshold determination. There is evidence to suggest that task difficulty might be affected by the differences in image statistics between $1 / f^{2}$ and $1 / f$ search areas. Eye movements are determined in part by the spatial properties typical of natural scenes (Nejemnik and Geisler 2005; Zhang et al 2008). Observers tend to avoid fixating in luminance patches of low spatial frequency (Tatler et al 2005). Specifically, differences in eye movements depending on the slope exponent of filtered noise patterns ( $\beta$ values) have been shown (Clarke et al 2008, 2009). In order to control for this effect, we first adjusted the contrast of the Gabor target for the $1 / f^{2}$ and $1 / f$ search areas so that the two conditions gave rise to visual searches of equivalent difficulty, based on individual performance. Additionally, eye movements were recorded to see if this could account for any potential findings. 


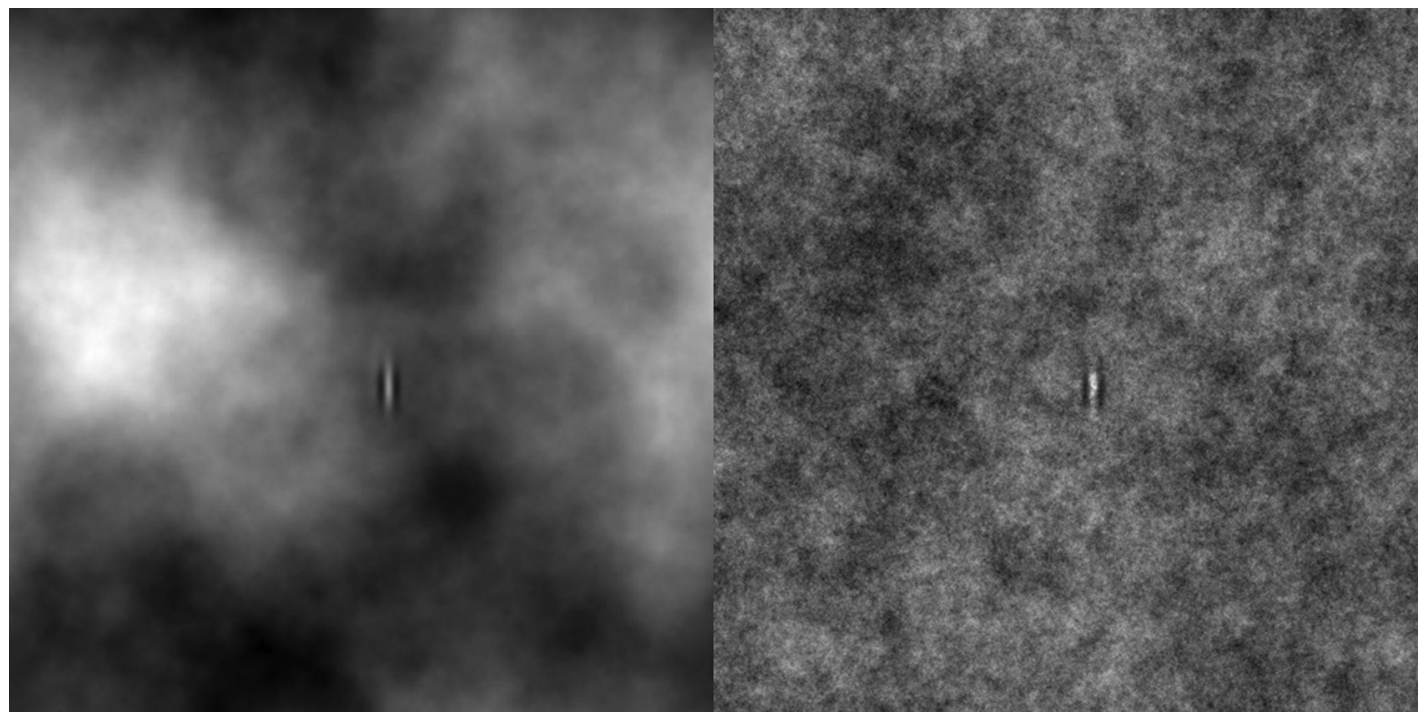

(a)

(b)

Figure 1. (a) Gabor target against $1 / f^{2}$ background; (b) Gabor target against $1 / f$ background. Target contrast is much higher in this demonstration than in the experiment, where it was determined individually for each observer.

Detection thresholds for the Gabor target against the $1 / f$ and $1 / f^{2}$ search backgrounds were determined in order to match stimuli for performance-defined difficulty in the visual search task. Stimuli were presented using the Psychtoolbox (Brainard 1997; Pelli 1997; Kleiner et al 2007) and the PEST staircase procedure (Taylor and Creelman 1967). Each individual trial commenced with a fixation cross against a mid-grey background. The stimulus consisted of a target presented against the relevant search background $\left(1 / f\right.$ or $\left.1 / f^{2}\right)$. The background type $(1 / f$ or $1 / f^{2}$ ) remained constant for the whole block. Noise was regenerated for each trial, thus no two noise patterns were identical, although their statistics were the same throughout the block. Half the observers started with the $1 / f$ background, the other half with the $1 / f^{2}$ background. The stimulus was presented for either a short $(2 \mathrm{~s})$ or long (10 s) duration. The 10 s condition was always completed first. In this part of the experiment the target was always present and the observer indicated which side of the screen the target appeared on using the arrow keys.

Two interleaved staircases tracking the contrast levels needed for $80 \%$ and $60 \%$ accuracy were conducted. The reversal rule is determined by a probability function that compares the likelihood of the current stimulus level being greater than the tracked probability (in this case $60 \%$ or $80 \%$ correct responses). The staircase step size was reduced by half on every reversal, and doubled on every third consecutive same response. Full details of the PEST procedure can be found in Taylor and Creelman (1967). A minimum of 150 trials were run in the slow presentation condition, and 300 in the fast presentation condition. The staircases were deemed to have converged within this number of trials, based on pilot data. The entire procedure was repeated for the comfortable and the uncomfortable backgrounds, and for the short $(2 \mathrm{~s})$ and long $(10 \mathrm{~s})$ presentation times.

Thresholds were obtained by fitting a logistic function using 'psignifit' (Fründ et al 2011). A bootstrapping procedure consisting of 999 simulations was implemented to estimate confidence intervals. The contrast level at which each observer performed at $75 \%$ correct detection was recorded as the threshold contrast level. For each observer a total of 4 thresholds were obtained: a short duration threshold and a long duration threshold, for the comfortable and uncomfortable stimuli. Thresholds from the short and long durations were then used to give "easy target" and "hard target" conditions in the visual search experiment below. 
2.1.5 Procedure: Visual search task. The results of the first part of the experiment were used to determine 4 stimulus contrast levels for each individual, based on performance, for each of the two search areas $\left(1 / f\right.$ and $\left.1 / f^{2}\right)$, and for each of the task difficulty levels (easy and hard). The observer's task in this second part of the experiment was to search for a small Gabor target hidden against either $1 / f$ or $1 / f^{2}$ filtered noise backgrounds. The target contrast was set individually for each observer at the $75 \%$ correct detection threshold from the first part of the experiment.

Stimuli were again presented at a random location on the screen, avoiding the central strip and the edges. This time, observers indicated whether the target was present or absent using the arrow keys on the keyboard. There were a total of 800 trials: 400 per condition, separated out into blocks of 100 trials each. The trial order was randomised. Approximately $30 \%$ of all trials were easy trials, with high-contrast targets. The easy (high-contrast target) trials were included for motivation and attention purposes: in low-prevalence search tasks there are many more misses than in high-prevalence search tasks (Judd et al 2011). Another approximately $30 \%$ were hard (low-contrast target) trials, with lower contrast targets. The final $40 \%$ of trials were target-absent trials. A fixation cross was presented on a mid-grey screen before the onset of each trial. Unlike the first part of the experiment, the second part was self-timed to an extent: observers initiated the trial with a button press, after which the stimulus was presented for a maximum of $10 \mathrm{~s}$. Observers were instructed to respond as quickly and as accurately as possible, within this time limit.

\subsection{Results}

2.2.1 Analysis. The percentage of correct trials was used as a measure of accuracy. Reaction times were log-transformed to normalise the distribution prior to statistical analysis. Analysis of eye movements was restricted to fixations longer than $0.2 \mathrm{~s}$ (Salthouse and Ellis 1980). One observer had to be excluded from the eye-movement analysis as eye movements were not recorded properly. However, behavioural results from this individual were still used. Log-transforms of the fixation duration and saccade length were used in the statistical analysis. Statistical analysis was conducted using IBM SPSS Statistics Version 19.

2.2.2 Visual search performance. The percentage of incorrect responses for each trial type (easy, hard, absent) is plotted on the right hand side of figure 2. Overall mean accuracy was $63.4 \%$ (standard deviation 14.7\%). Mean reaction times are plotted on the left hand side of figure 2.

In order to check whether our contrast matching manipulation was successful, we performed an analysis on the target-present trials. Bartlett's Test showed there to be unequal variances $\left(\chi_{2}^{2}=30.12, p<0.01\right)$. As expected, results of a 2 (background) $\times 2$ (target difficulty) repeatedmeasures MANOVA showed there to be an effect of target difficulty (easy or hard) only $\left(F_{2,6}=42.77, p<0.01\right)$. From figure 2 it can be seen that RTs are shorter on easy trials, then hard trials, then target-absent trials. There are also fewer incorrect responses for easy trials than for hard and target-absent trials. There is no difference between the two background types.

To address our specific hypothesis, results of a repeated-measures MANOVA showed there to be no effect of background on either reaction time or accuracy for target-absent trials $\left(F_{2,6}=2.06, p=\mathrm{ns}\right)$.

2.2.3 Eye movements. The results of two-tailed repeated-measures $t$-tests showed there to be no statistically significant differences between either mean fixation duration $\left(1 / f^{2}\right.$ mean $=0.49 \mathrm{~s}$, $1 / f$ mean $=0.53 \mathrm{~s})$ or mean saccade length $\left(1 / f^{2}\right.$ mean $=259.03,1 / f$ mean $=235.43$ pixels $)$ between the two search areas. (Fixation duration: $t_{6}=-2.69, p=\mathrm{ns}$; saccade length, $t_{6}=0.48$, $p=$ ns.) 


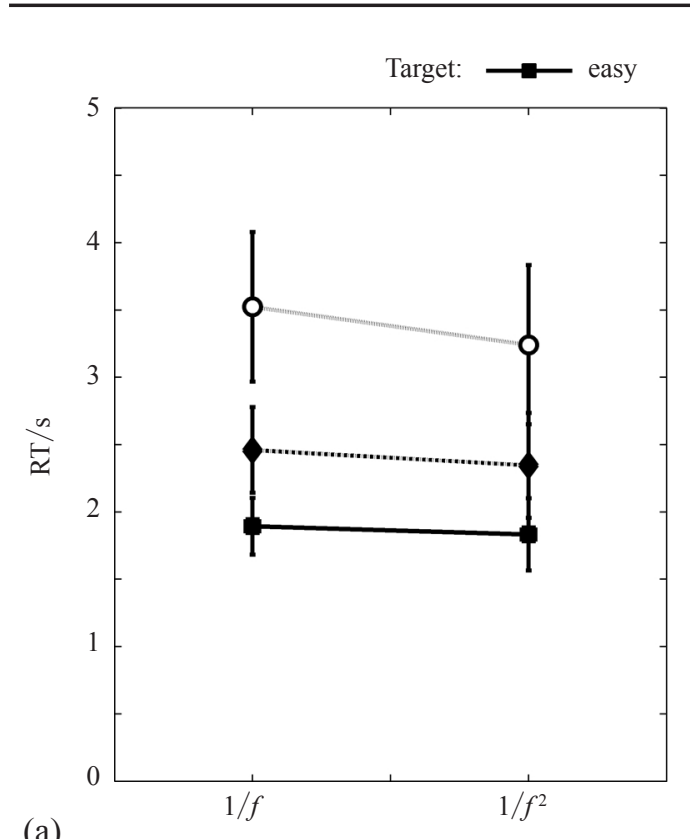

(a)

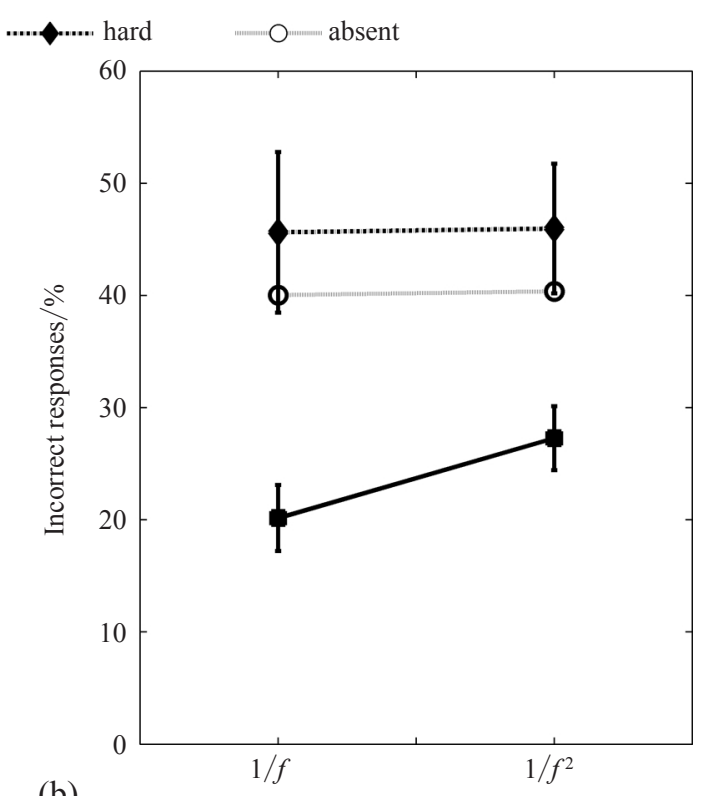

Figure 2. Reaction time (a) and percentage of incorrect responses (b) as a function of the background type. Results are plotted separately for easy, hard, and target-absent trials. Error bars in this and all other figures show \pm 1 standard error.

\subsection{Discussion}

Experiment 1 was conducted to ascertain whether there were differences in performance in a visual search task against $1 / f^{2}$ and $1 / f$ backgrounds, which have been shown in previous research to influence judgments of discomfort (Juricevic et al 2010). The results of experiment 1 show no evidence that observers shifted criterion in order to avoid prolonged exposure to stimuli previously judged to be uncomfortable. As there was no effect of background on the target-present trials, this shows that the difficulty matching part of the experiment was successful. As expected, there was an effect of target type: easier trials were found more quickly and more accurately than hard trials. However, for our specific prediction, there was no effect of background on target-absent trials, indicating search motivation was not influenced by task difficulty. The hard trials showed near-chance accuracy, which could potentially create a floor effect, and account for the lack of difference between the backgrounds for the hard trials. However, as the easy trials did not show this potential floor effect, this explanation cannot account for the lack of effects of background on performance. Also no differences were found between the eye-movement behaviour against the two search areas.

One possible reason that the type of background had no effect on performance is that the stimuli were simply not sufficiently uncomfortable to elicit detriments to performance. Therefore, in the next experiment we investigated the effects of striped patterns on performance, using the same visual search task.

\section{Experiment 2. Striped backgrounds}

Previous research has shown striped patterns to be uncomfortable (Wilkins et al 1984; Wilkins 1995), and to be capable of inducing epileptiform EEG patterns of activation in epilepsy sufferers (Wilkins et al 1979), and even epileptic seizures in those with pattern-sensitive epilepsy (Radhakrishnan et al 2005). Therefore, the study was extended to investigate the effects of striped gratings on visual search performance, as these are expected to be more uncomfortable than the noise patterns. 
Previous work on both clinical and general populations has shown spatial frequency tuning in the discomfort of striped patterns (Wilkins et al 1979, 1984). If any effects on accuracy found are due to discomfort, we would expect them to be similarly spatial frequency tuned.

The search strategy of observers is very task-dependent (Boot et al 2009). Therefore, in order to be able to compare between experiments, the search task remained the same: the Gabor target was hidden against a filtered noise search area. However, this time the search was limited to a central filtered noise search area with the rest of the display consisting of an outer background. This method also avoided the performance-matching concerns that were evident in experiment 1.

\subsection{Method}

3.1.1 Apparatus. The apparatus used was the same as in experiment 1 .

3.1.2 Stimuli. The target was located within a central circular patch consisting of $1 / f$ filtered noise. The central circular region of the patch subtended a visual angle of $6.05 \mathrm{deg}$, and had a Gaussian smoothed edge ( $\sigma=1.97 \mathrm{deg}$ ). In condition (i), the target was the same 3 cycles deg ${ }^{-1}$ Gabor as before; in condition (ii), the target was a 0.75 cycle deg $^{-1}$ Gabor. The contrast of the target was fixed to be either $20 \%$ (hard trials) or $30 \%$ (easy trials). Example stimuli can be seen in figure 3 .

The background outside the central patch was manipulated: either the background was uniform mid-grey, or a vertical sinewave grating of $0.75,1.5$, or 3 cycles $\mathrm{deg}^{-1}$, at $50 \%$ contrast.

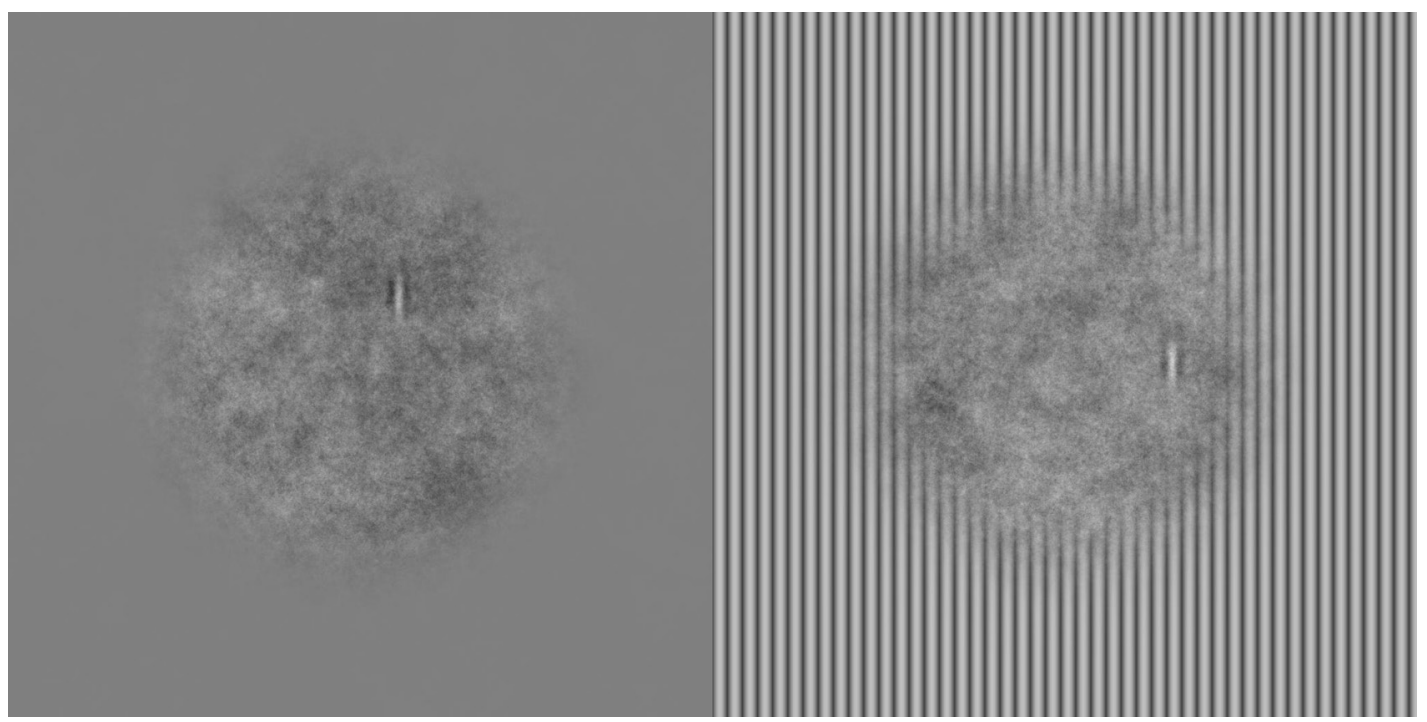

Figure 3. Example stimuli for experiment 2. The target Gabor was always in the central $1 / f$ noise patch. The outer background (mid-grey or striped) was manipulated between blocks.

3.1.3 Observers. Twenty-four observers participated in the study, twelve in condition (i), twelve in condition (ii) (mean $=22.5$ years, $\mathrm{SD}=1.86$ years). All were naive to the purposes of the experiment.

3.1.4 Procedure. There were 4 blocks ( 1 for each spatial frequency of the outer background) of 100 trials each per condition (mid-grey or striped outer background). Five observers in condition (i) completed the experiment with 200 trials in each block. The rest all completed 100 trials per block. Reducing the number of trials had no effect on the mean reaction times or accuracies unless fewer than 50 trials were incorporated. Stimuli were displayed for a 
maximum of $10 \mathrm{~s}$. Observers were asked to search for the Gabor target and respond as quickly and accurately as possible using the left (target present) or right (target absent) arrow keys, within the time limit. Participants were informed that the target might be absent, and that the target (if present) would always be located in the central noise pattern. Each trial was initiated by the observers themselves using the down arrow.

\subsection{Results}

Figure 4 shows the results for the 3 cycles deg ${ }^{-1}$ target. The left hand side of figure 4 shows mean reaction time plotted against the spatial frequency of the background, for easy, hard, and target-absent trials. Reaction time tends to increase with trial difficulty (easy trials have the shortest time), and also with spatial frequency. The right hand side of figure 4 shows the mean inaccuracy, against the spatial frequency of the background, for easy, hard, and target-absent trials. Again, worse performance (more inaccuracy) is shown with increasing spatial frequency, except for the target-absent trials. There are, however, very few inaccurate responses for target-absent trials overall.

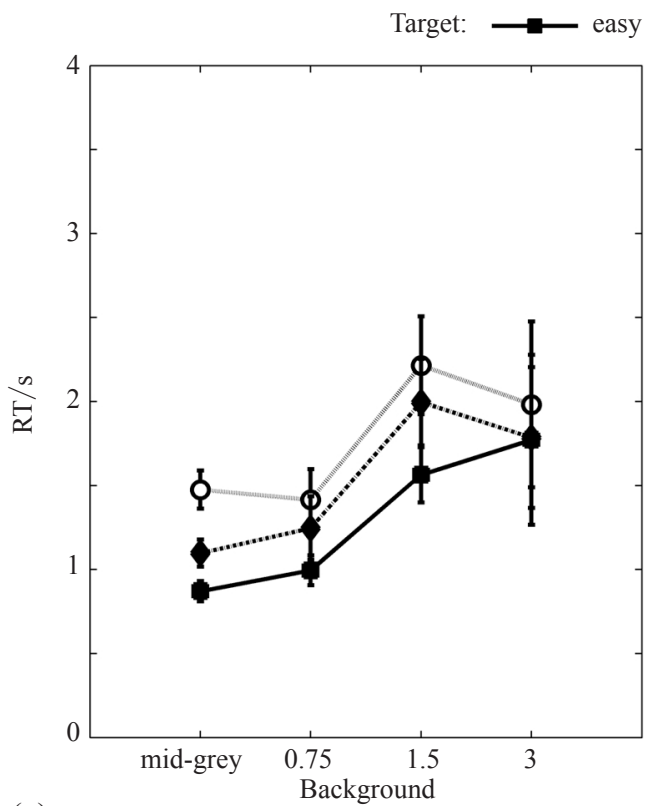

(a)

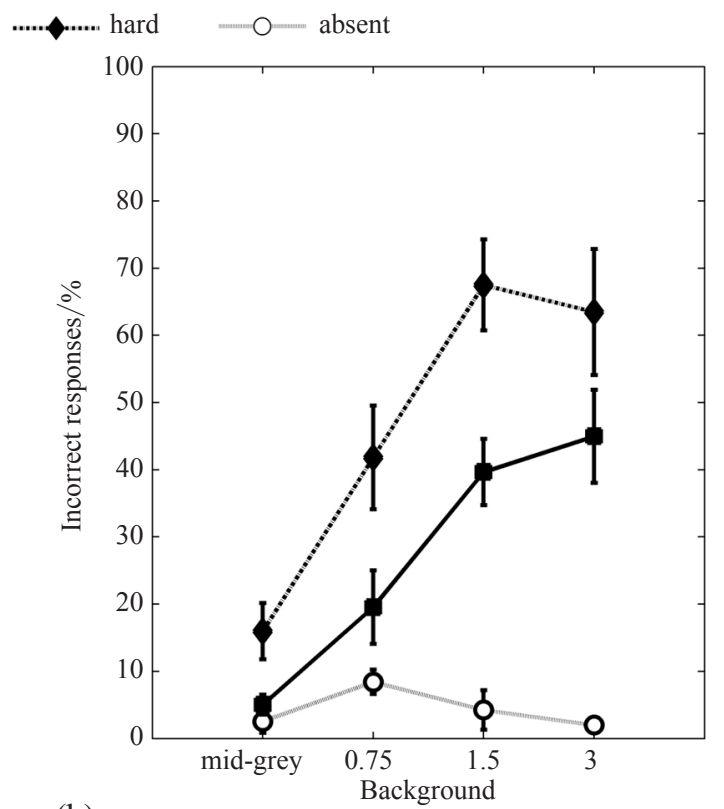

(b)

Figure 4. Mean reaction time (a) and percentage inaccuracy (b) against spatial frequency of background with a 3 cycles deg ${ }^{-1}$ target.

The results of a 3 (trial type) $\times 4$ (background) repeated-measures MANOVA showed there to be a significant effect of trial type (easy, hard, absent) on reaction time and accuracy $\left(F_{4,44}=30.99, p<0.01\right)$. There was also a main effect of background spatial frequency on reaction time and accuracy $\left(F_{4,66}=6.76, p<0.01\right)$. There was an interaction effect of trial type and background on reaction time and accuracy $\left(F_{12,132}=7.33, p<0.01\right)$. Univariate follow-up tests showed that there was a significant main effect of trial type on both accuracy and reaction time $\left(F_{2,22}=53.90, p<0.01 ; F_{2,22}=35.70, p<0.01\right)$. There was a significant main effect of background on both accuracy and reaction time $\left(F_{2.2,24.4}=21.0, p<0.01\right.$; $\left.F_{3,33}=4.75, p<0.01\right)$. There was a significant trial type $\times$ background interaction effect on accuracy $\left(F_{3.2,35.7}=13.85, p<0.01\right)$ and on reaction time $\left(F_{6,66}=7.78, p<0.01\right)$.

Results of a posteriori repeated-measures $t$-tests show that, for the $3 \mathrm{cycles} \mathrm{deg}^{-1}$ target, observers were significantly less accurate when the background was also $3 \mathrm{cycles} \mathrm{deg}^{-1}$ than when it was $0.75 \mathrm{cycle} \mathrm{deg}^{-1}$, for the target absent, easy, and hard trials, respectively $\left(t_{11}=3.03\right.$, 
$\left.p<0.01 ; t_{11}=4.49, p<0.01 ; t_{11}=3.54, p<0.01\right)$. There was also a significant difference in the reaction times for easy and hard trials $\left(t_{11}=2.55, p<0.05 ; t_{11}=2.64, p<0.05\right)$, but no significant difference for the target-absent reaction times $\left(t_{11}=1.31, \mathrm{~ns}\right)$.

Figure 5 shows the results for the 0.75 cycle deg $^{-1}$ target. The left-hand side of figure 5 shows reaction time, the right-hand side shows inaccuracy against spatial frequency of the background for the three levels of task difficulty (easy, hard, absent). There is an apparent effect of trial type on both reaction time and accuracy. There are only a few incorrect responses for the target-absent trials. There is an effect of spatial frequency, although in comparison with the results for the 3 cycles deg ${ }^{-1}$ target, the spatial frequency tuning has shifted-worst performance is now found for the $0.75 \mathrm{cycle} \mathrm{deg}^{-1}$ background, both in terms of reaction times and accuracy.

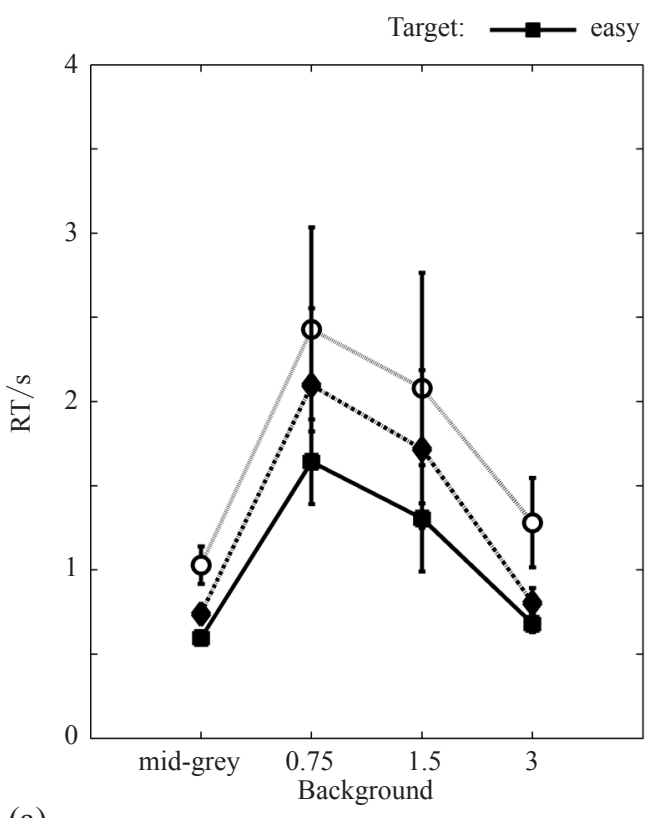

(a)

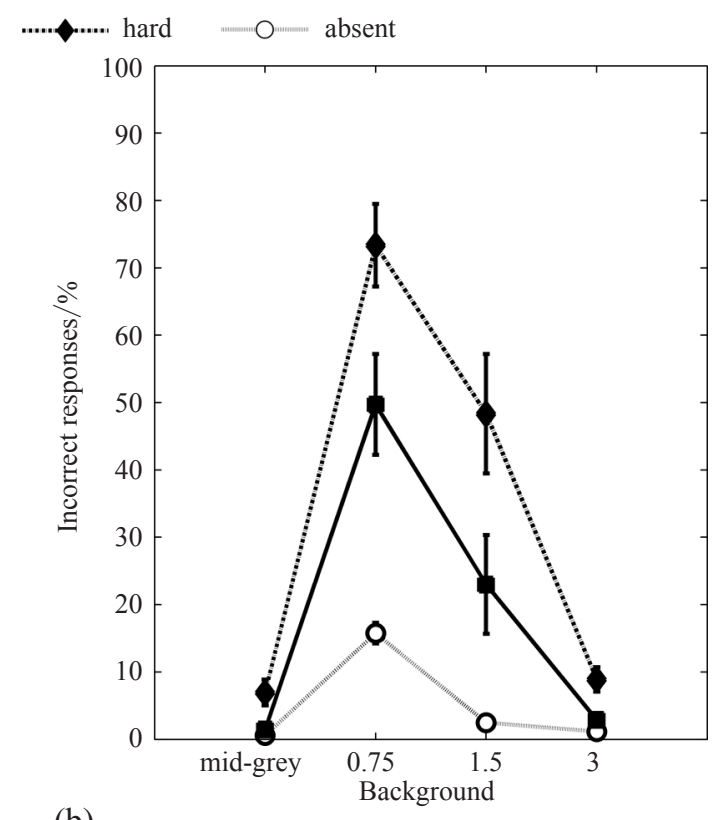

(b)

Figure 5. Mean reaction time (a) and percentage inaccuracy (b) against spatial frequency of background with a 0.75 cycle deg $^{-1}$ target.

The results of a 3 (trial type) $\times 4$ (background) repeated-measures MANOVA showed there to be a significant effect of trial type (easy, hard, absent) on reaction time and accuracy $\left(F_{4,44}=20.76, p<0.01\right)$, and also a main effect of background spatial frequency on reaction time and accuracy $\left(F_{6,66}=13.72, p<0.01\right)$. There was also an interaction effect of trial type and background on reaction time and accuracy $\left(F_{12,132}=15.0, p<0.01\right)$. Univariate follow-up tests showed that there was a significant main effect of trial type on both accuracy and reaction time $\left(F_{2,22}=61.34, p<0.01 ; F_{2,22}=14.37 p<0.01\right)$. There was a significant main effect of background on both accuracy and reaction time $\left(F_{3,33}=37.66, p<0.01\right.$; $\left.F_{3,33}=14.92, p<0.01\right)$. There was a significant effect of the trial type $\times$ background on accuracy $\left(F_{6,66}=17.92, p<0.01\right)$ and on reaction time $\left(F_{6,66}=12.67, p<0.01\right)$.

Results of a posteriori repeated-measures $t$-tests show that for the $0.75 \mathrm{cycle} \mathrm{deg}^{-1}$ target observers were both significantly less accurate and slower when the background was also 0.75 cycle deg $^{-1}$ than when it was 3 cycles deg $^{-1}$, for the target absent, easy, and hard trials, respectively $\left(t_{11}=9.79, p<0.01 ; t_{11}=6.39, p<0.01 ; t_{11}=10.30, p<0.01\right)$. There was also a significant difference for the reaction times $\left(t_{11}=3.60, p<0.05 ; t_{11}=3.70, p<0.01\right.$; $\left.t_{11}=5.65, p<0.01\right)$. This can be seen in figure 5: tuning in both reaction time and accuracy

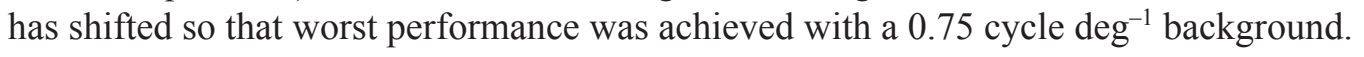




\subsection{Discussion}

The results of experiment 2 show that there were significant main effects of trial type and outer background spatial frequency on accuracy and reaction time at finding a Gabor target hidden against a central $1 / f$ noise patch. The effect of trial type was expected: performance is increased on high-contrast, easy trials. The main effect of background is more interesting, and can be seen in the spatial frequency tuning effects in figures 4 and 5. Poorest performance, both in terms of accuracy and reaction time, is seen when the spatial frequency of the target is similar to that of the background.

The presence of any sinusoidal grating background resulted in worse performance than with a mid-grey background. This could be explained as being due to the discomfort that has been previously reported for striped patterns (Wilkins et al 1984). The most uncomfortable spatial frequencies have been indicated as being around 4 cycles $\mathrm{deg}^{-1}$ for striped patterns (Wilkins et al 1984). Spatial frequencies around 3 cycles deg-1 have previously been suggested as those resulting in maximum discomfort (Fernandez and Wilkins 2008). Spatial frequency tuning was also found in discomfort judgments of filtered noise patterns (O'Hare and Hibbard 2011). Therefore, if discomfort was the reason for the detriment to performance, it is expected that worst performance would be around $3 \mathrm{cycles} \mathrm{deg}^{-1}$. However, the results show that the spatial frequency tuning depends on the spatial frequency of the target, such that worst performance was found when the spatial frequencies of the target and background were matched. This suggests that a large proportion of the decrement in performance results from the similarity between the spatial frequency of the background and the target, rather than the spatial frequency of the background per se. Therefore, the detriment in performance could be due to other effects, such as surround suppression. Surround suppression is a reduction in perceived contrast of a target caused by the presence of the peripheral background, possibly resulting from receptive field architecture and the effects of inhibitory interneurons (eg Webb et al 2005). Surround suppression effects are greatest when target and background are of the same spatial frequency and orientation, and increase with eccentricity (Petrov et al 2005). Maximum effects of surround suppression are seen when the target and the background are at around $10 \%$ contrast, and are also important in the range of contrasts used in the current experiment (Petrov et al 2007).

A final experiment was therefore conducted in which observers searched for a horizontal target, in the presence of a vertical background. If the results are due to discomfort rather than surround suppression, then there should be a residual effect when the target is orthogonal to the stripes.

\section{Experiment 3. The effect of target orientation}

\subsection{Method}

4.1.1 Apparatus, stimuli, and procedure. They were the same as in experiment 2. Only one target was used, a horizontal 3 cycles deg $^{-1}$ Gabor patch.

4.1.2 Observers. Thirteen observers took part in this study. This included two observers, who had previously participated in experiment 2 and returned to participate in this part of the study. Two of the authors ( $\mathrm{LOH}$ and $\mathrm{PBH})$, who are experienced psychophysical observers with corrected-to-normal vision, and an additional nine other naive participants also participated in the study (mean age $=23.92$ years, $\mathrm{SD}=5.01$ years).

\subsection{Results}

A $3 \times 4$ way repeated-measures MANOVA was conducted to assess the effects of the trial type and background on accuracy and noise. Bartlett's test showed there to be unequal variances $\left(\chi_{2}^{2}=23.32, p<0.01\right)$. The only significant multivariate effect was that of trial 
type $\left(F_{4,48}=15.47, p<0.01\right)$. Therefore, there was no effect of background on accuracy or reaction time when the target was orthogonal to the background. This can be seen in figure 6: there is a clear lack of spatial frequency tuning, indicating no effect of background on the reaction times or the accuracy.
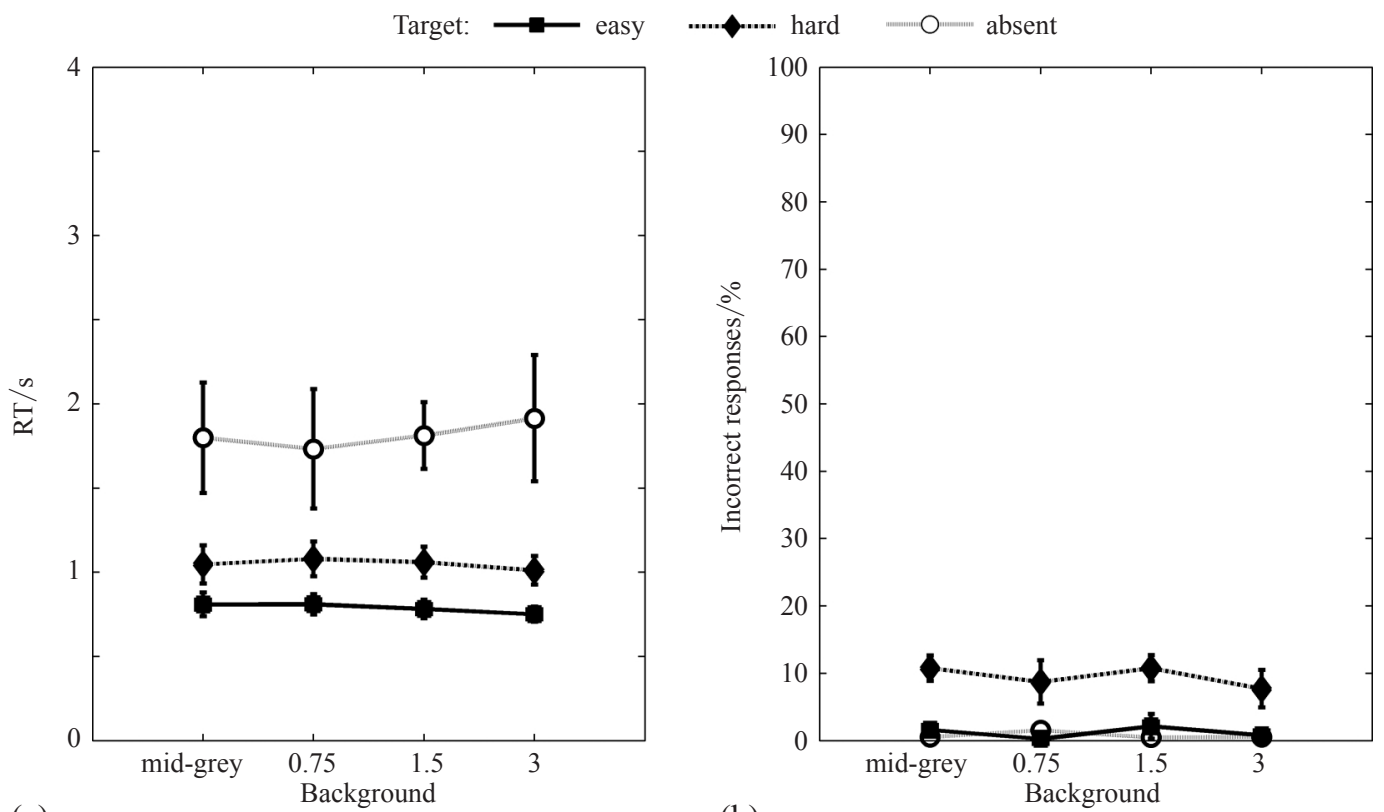

(a)

(b)

Figure 6. Mean reaction time (a) and percentage inaccuracy (b) as a function of background spatial frequency, with a horizontally oriented Gabor target.

\subsection{Discussion}

The results of experiment 3 showed that the tuning effect found in experiment 2 disappeared when the target (a Gabor patch) was at an orthogonal orientation to the outer background stripes. This suggests that the tuning found previously can be explained by surround suppression effects alone, and no effect can be attributed to visual discomfort in the current task.

\section{Conclusion}

Experiment 1 investigated the possibility that uncomfortable filtered noise search backgrounds have a detrimental effect on visual search tasks, when efforts were made to match stimuli for task difficulty. The critical comparison was the target-absent trials-it was thought that observers would quit searching faster against more uncomfortable backgrounds (thought to be $1 / f^{2}$ ). Our results did not support this hypothesis: there was no evidence of a difference between the 2 search backgrounds in terms of reaction time, accuracy, or eye movements.

In experiment 2 , the search task remained the same, but this time an outer task-irrelevant background was introduced: either mid-grey or striped gratings of varying spatial frequency. It was predicted that, if there was a substantial amount of discomfort, then there will be a performance detriment that should follow the spatial frequency tuning previously shown in the discomfort literature (Wilkins et al 1984).

The results of experiment 2 showed a tuning effect on reaction time and accuracy of search for outer backgrounds of different spatial frequencies. There was a reduction in performance with any of the striped outer backgrounds compared to the mid-grey outer background: both longer reaction times and reduced accuracy. This suggests that any modulation in the background causes a detriment to performance. However, performance was worst when the spatial frequency of the target matched that of the background. As this 
tuning did not follow the theoretically most uncomfortable background spatial frequency, but instead shifted depending on the target, this suggests the effect is unrelated to discomfort, and possibly resulting from alternative processes such as surround suppression (Petrov et al 2005). This explanation was explored in experiment 3 , in which the orientation of the Gabor target was orthogonal to the striped outer background. Under these conditions no effect of background was observed. This suggests that there are no effects of discomfort on visual search performance in the present experiment.

Effects of discomfort on visual tasks have been previously demonstrated in non-clinical populations by both Conlon et al (1998) and Chronicle and Wilkins (1996). One important difference is that observers were searching for targets in a striped background in the Conlon et al search task, whereas in the current situation the background and the target were displaced using the filtered noise search area. The Chronicle and Wilkins task involved detection of a letter directly against the striped background. Spatial frequency of the target was matched to the background. Thus the lack of an effect on performance in the current study could be because the target was not directly against the stripes, and this would reduce discomfort effects (Wilkins et al 1984). Therefore discomfort might have been weaker than in previous investigations.

In summary, this study shows that theoretically uncomfortable stimuli did not affect performance on a visual search task in a non-clinical population. Thus, if search strategy was altered by differences in motivation due to discomfort, this effect was small in comparison to interference effects. This suggests that, although non-clinical populations can judge images for discomfort (Juricevic et al 2010), these discomfort effects are not substantial enough to affect performance. More extreme discomfort responses that are powerful enough to manifest themselves in performance measures might be seen in clinical populations. However, investigating the differences between populations is not the objective of this study and thus remains for future research.

This is not to say, however, that global image properties are not an important factor that affects visual performance, and indeed such effects are reported here and elsewhere. Rather, we argue that the degree of similarity between the target of interest and the background is an important determinant of the level of disruption of performance that will occur. In many everyday tasks, such as reading, the target and the background will show many visual similarities. This might then be reflected in the disruption to performance in some studies (eg Allen et al 2008).

\section{References}

Allen P W, Gilchrist J M, Hollis J, 2008 "Use of visual search in the assessment of pattern-related visual stress (PRVS) and its alleviation by coloured filters" Investigative Ophthalmology \& Visual Science $494210-4218$

Aurora S K, Wilkinson F, 2007 "The brain is hyperexcitable in migraine" Cephalalgia 27 1422-1435

Barlow H B, 1961 "Possible principles underlying the transformations of sensory messages", in Sensory Communication Ed. W A Rosenblith (London: John Wiley and Sons) pp 217-234

Boot W R, Becic E, Kramer A F, 2009 "Stable individual differences in search strategy? The effect of task demands and motivational factors on scanning strategy in visual search" Journal of Vision 9(3):7, 1-16

Brainard D H, 1997 “The Psychophysics Toolbox" Spatial Vision 10 433-436

Chase C, Tosha C, Borsting E, Ridder W H III, 2009 "Visual discomfort and objective measures of static accommodation" Optometry and Vision Science 86 883-889

Chronicle E P, Wilkins A J, 1996 "Gratings that induce perceptual distortions mask superimposed targets" Perception 25 661-668

Clarke A D F, Green P R, Chantler M J, Emrith K, 2008 "Visual search for a target against a $1 / f^{\beta}$ continuous textured background" Vision Research 48 2193-2203 
Clarke A D F, Chantler M J, Green P R, 2009 "Modelling visual search on a rough surface" Journal of Vision 9(4): 11, 1-12

Conlon E G, Hine T, 2000 "The influence of pattern interference on performance in migraine and visual discomfort groups" Cephalalgia 20 708-713

Conlon E G, Humphreys L, 2001 "Visual search in migraine and visual discomfort groups" Vision Research 41 3063-3068

Conlon E G, Lovegrove W, Barker S, Chekaluk E, 2001 "Visual discomfort: the influence of spatial frequency" Perception 30 571-581

Conlon E G, Lovegrove W J, Chekaluk E, Pattison P E, 1999 "Measuring visual discomfort" Visual Cognition 6 637-663

Conlon E G, Lovegrove W, Hine T, Chekaluk E, Piatek K, Hayes-Williams K, 1998 "The effects of visual discomfort and pattern structure on visual search" Perception 27 21-23

Cornelissen F W, Peters E, Palmer J, 2002 "The Eyelink Toolbox: Eye tracking with MATLAB and the Psychophysics Toolbox" Behavior Research Methods, Instruments, \& Computers 34 613-617

Fernandez D, Wilkins A J, 2008 "Uncomfortable images in art and nature" Perception 37 1098-1113

Field D J, 1987 "Relations between the statistics of natural images and the response properties of cortical cells" Journal of the Optical Society of America A 4 2379-2394

Field D J, 1994 "What is the goal of sensory coding?" Neural Computation 6 559-601

Fründ I, Haenel N V, Wichmann F A, 2011 "Inference for psychometric functions in the presence of non-stationary behaviour" Journal of Vision 11(6):16, 1-19

Judd T, Durand F, Torralba A, 2011 "Fixations on low-resolution images" Journal of Vision 11(4): 14, $1-20$

Juricevic I, Land L, Wilkins A, Webster M, 2010 "Visual discomfort and natural image statistics" Perception 39 884-899

Kleiner M, Brainard D, Pelli D, 2007 "What's new in Psychtoolbox-3?" Perception 36 ECVP Supplement, 14

Lambooij M, Isselsteijn W A, Heynderickx I, 2011 "Visual discomfort of 3D TV: Assessment methods and modelling" Displays 5 209-218

Leat S, Li W, Epp K, 1999 "Crowding in central and eccentric vision: The effects of contour interaction and attention" Investigative Ophthalmology \& Visual Science 40 504-512

Lichtenstein-Vidne L, Henik A, Safadi Z, 2007 "Task-relevance modulates the effects of peripheral distractors" Quarterly Journal of Experimental Psychology 60 1216-1226

Marcus D A, Soso M J, 1989 "Migraine and stripe-induced visual discomfort" Archives of Neurology 46 1129-1132

Nejemnik J, Geisler W S, 2005 "Optimal eye movement strategies in visual search" Nature 434 387-391

O'Hare L, Hibbard P B, 2011 "Spatial frequency and visual discomfort" Vision Research 51 1767-1777

Pelli D G, 1997 "The VideoToolbox software for visual psychophysics: Transforming numbers into movies" Spatial Vision 10 437-442

Petrov Y, Carandini M, McKee S, 2005 "Two distinct mechanisms of surround suppression in human vision" Journal of Neuroscience 25 8704-8707

Petrov Y, Popple A V, McKee S P, 2007 "Crowding and surround suppression: Not to be confused" Journal of Vision 7(2):12, 1-9

Radhakrishnan K, St Louis E K, Johnson J A, McClelland R L, Westmoreland B F, Klass D W, 2005 "Pattern-sensitive epilepsy: Electroclinical characteristics, natural history and delination of the epileptic syndrome" Epilepsia $\mathbf{4 6}$ 48-58

Salthouse T A, Ellis C L, 1980 "Determinants of eye-fixation duration" American Journal of Psychology 92 207-234

Sheedy J E, Hayes J, Engle J, 2003 "Is all aesthenopia the same?" Optometry and Vision Science $80732-739$

Simoncelli E P, Olshausen B A, 2001 "Natural image statistics and neural representation" Annual Review of Neuroscience 24 1193-1216

Tatler B W, Baddeley R J, Gilchrist I D, 2005 "Visual correlates of fixation selection: Effects of scale and time" Vision Research 45 643-659 
Taylor M M, Creelman C D, 1967 "PEST: Efficient estimates on probability functions" Journal of the Acoustical Society of America 74 1367-1374

Tolhurst D J, Tadmor Y, Chao T, 1992 “Amplitude spectra of natural images” Ophthalmological and Physiological Optics 12 229-232

Webb B S, Dhruv N T, Solomon S G, Tailby C, Lennie P, 2005 "Early and late mechanisms of surround suppression in the striate cortex of the macaque" Journal of Neuroscience 25 11666-11675

Wilkins A J, 1995 Visual Stress (Oxford: Oxford University Press)

Wilkins A J, Andermann F, Ives J, 1975 "Stripes, complex cells and seizures: An attempt to determine the locus and nature of the trigger mechanism in pattern sensitive epilepsy" Brain 98 365-380

Wilkins A J, Darby C E, Binnie C D, 1979 "Neurological aspects of pattern-sensitive epilepsy" Brain $1021-25$

Wilkins A J, Nimmo-Smith I, Tait A, McManus C, Della Sala S, Tilley A, Arnold K, Barrie M, Scott S, 1984 "A neurological basis for visual discomfort" Brain 107 989-1017

Zhang L, Tong M H, Marks T K, Shan H, Cottrell G W, 2008 "SUN: A Bayesian framework for saliency using natural statistics" Journal of Vision 8(7):32, 1-20 